\title{
Relocalization of cell adhesion molecules during neoplastic transformation of human fibroblasts
}

\author{
CRISTINA BELGIOVINE, ILARIA CHIODI and CHIARA MONDELLO \\ Istituto di Genetica Molecolare, Consiglio Nazionale delle Ricerche, Via Abbiategrasso 207, 27100 Pavia, Italy
}

Received May 6, 2011; Accepted June 10, 2011

DOI: 10.3892/ijo.2011.1119

\begin{abstract}
Studying neoplastic transformation of telomerase immortalized human fibroblasts (cen3tel), we found that the transition from normal to tumorigenic cells was associated with the loss of growth contact inhibition, the acquisition of an epithelial-like morphology and a change in actin organization, from stress fibers to cortical bundles. We show here that these variations were paralleled by an increase in $\mathrm{N}$-cadherin expression and relocalization of different adhesion molecules, such as $\mathrm{N}$-cadherin, $\alpha$-catenin, $\mathrm{p}-120$ and $\beta$-catenin. These proteins presented a clear membrane localization in tumorigenic cells compared to a more diffuse, cytoplasmic distribution in primary fibroblasts and non-tumorigenic immortalized cells, suggesting that tumorigenic cells could form strong cell-cell contacts and cell contacts did not induce growth inhibition. The epithelial-like appearance of tumorigenic cells did not reflect a mesenchymal-epithelial transition; in fact, cen3tel cells expressed vimentin and did not express cytokeratins at all transformation stages. Moreover, they did not express epithelial proteins such as occluding and claudin-1. In contrast, ZO-1 showed higher levels and a more defined membrane localization in tumorigenic cells compared to non-tumorigenic cells; this confirms its role in adherens junction formation in mesenchymal cells and is in agreement with the strong cellcell contact formation by neoplastically transformed cells Finally, we found $\alpha$-catenin and ZO-1 nuclear localization in non-transformed cells, suggestive of possible additional roles of these proteins besides cell junction formation.
\end{abstract}

\section{Introduction}

Neoplastic transformation involves changes in a great variety of cellular functions, among which cell adhesion. Cadherins and catenins play essential roles in the establishment of

Correspondence to: Dr Chiara Mondello, Istituto di Genetica Molecolare, Consiglio Nazionale delle Ricerche, Via Abbiategrasso 207, 27100 Pavia, Italy

E-mail: mondello@igm.cnr.it

Key words: neoplastic transformation, human fibroblasts, cadherin, catenin, tight junctions, vimentin, cytokeratin, ZO-1 cell-cell contacts $(1,2)$. Cadherins are transmembrane glycoproteins mediating homotypic cell-cell adhesion via their extracellular domain. Through their cytoplasmic domain, they bind to catenins, which mediate the connection with the actin cytoskeleton. Different types of cadherins are expressed in different cell types; e.g. N-cadherin is typically expressed in mesenchymal cells, such as fibroblasts, while E-cadherin participates in the formation of adherens junctions in cells of epithelial origin. The role of E-cadherin and $\beta$-catenin in the development and progression of tumors of epithelial origin is well documented (3). In particular, loosening of cell-cell contacts because of loss of E-cadherin expression and nuclear accumulation of $\beta$-catenin are hallmarks of the epithelialmesenchymal transition (EMT) (4), which is associated with progression towards the metastatic phenotype in different types of carcinomas. During EMT, a switch in the type of intermediate filaments from cytokeratins to vimentin is generally observed, together with a change from circumferential F-actin fibers to a network of stress fibers.

An opposite transition, from mesenchymal to epithelial phenotype (MET; mesenchymal-epithelial transition) occurs in some mesenchymal tumors. Glandular epithelial differentiation was described in some synovial sarcomas and, rarely, in some mesenchymal bone neoplasms (5). MET is generally associated with up-regulation of E-cadherin and expression of cytokeratins. Expression of tight junction proteins, which are typical of epithelial cells, with formation of well-organized junction structures, was detected in glands of synovial sarcomas, suggesting that true epithelial differentiation occurs in these tumors (6).

By ectopic telomerase expression, we immortalized a human fibroblast cell line. During culture propagation, immortalized cells, named cen3tel, underwent neoplastic transformation, they became able to induce tumors when subcutaneously injected into nude mice and then acquired metastatic capacity (7-9). Cen3tel cells at the early stages of tumorigenicity formed pleomorphic sarcomas in nude mice, cells at later stages induced tumors with a hemangioperycitoma-like vascular pattern, analogous to that observed in synovial sarcomas. In cen3tel cells, neoplastic transformation was associated with an increase in cell growth rate, the loss of contact inhibition and the achievement of a high cellular density. Cells changed morphology, from the typical fibroblastic elongated shape to a polygonal one, and actin organization, from stress fibers to cortical rings, similar to that of epithelial cells $(7,9)$. Moreover, 
tumorigenic cells showed increased invasiveness, which did not depend on matrix-protease activity, as expected for cells of mesenchymal origin, but on the RhoA-dependent kinase ROCK, as described for cells using the amoeboid movement (7,10-12).

Given the complex morphological and functional changes observed during neoplastic transformation of cen3tel cells, we aimed at analyzing whether they were associated with variations in expression and cellular localization of cadherins and catenins, together with that of mesenchymal and epithelial markers.

\section{Materials and methods}

Cells and cell culture. The cen3tel telomerase immortalized cell line was obtained from primary cen 3 fibroblasts, derived from a centenarian individual, by infection with an hTERTcontaining retrovirus (8). Cen3tel cells were used at different steps of propagation (up to around PD 1000) reflecting different phases of transformation (7-9). Early cen3tel cells were at the initial passages after the infection with the hTERT-containing retrovirus (between PDs 34 and 45) and behaved similarly to primary cen 3 fibroblasts; mid cen3tel cells (around PD 100) were at an early phase of transformation, they grew in the absence of solid support, lost contact inhibition, but were not tumorigenic in nude mice; tumorigenic cen3tel cells, which induced tumors when subcutaneously inoculated in nude mice, were further subdivided in three groups, according to the time required for tumor formation, which decreased at increasing PDs: tumorigenic cells of phase I (around PD 160), phase II (around PD 600) and phase III (around PD 1000). Phase III tumorigenic cells displayed also metastatic capacity (7). Primary, immortalized and SW480 cells were grown in Dulbecco's modified Eagle's medium (DMEM, Celbio) supplemented with $10 \%$ fetal bovine serum (Lonza).

Antibodies. The following primary antibodies were used: anti-N-cadherin (clone 32), anti-p120 (clone 98) (both from BD Transduction Laboratories Biosciences); anti- $\beta$-catenin (MAB2081, Chemicon); anti- $\alpha$-catenin (C2081, Sigma-Aldrich); anti-vimentin (S-20, SC-7558, Santa Cruz); anti-cytokeratin (KL1, Immunotech); anti-ZO-1 (cat. no. 33-9100), anti-claudin-1 (cat.no.33-1500), anti-occludin (cat.no. 51-9000) (all fromZymed Laboratories); anti- $\gamma$ tubulin (clone GTU-88, Sigma-Aldrich). Secondary antibodies, either conjugated with a fluorochrome (for immunofluorescence) or with horseradish peroxidase (for Western blotting) were from Jackson Immunoresearch.

Indirect immunofluorescence. Cells were seeded in 12-well plates (Corning) containing $19 \mathrm{~mm}$ diameter coverslips and incubated at $37^{\circ} \mathrm{C}$ for $48 \mathrm{~h}$ to allow cells to form a high density monolayer. For detection of vimentin, cytokeratins, $\mathrm{N}$-cadherin, p120, $\beta$-catenin and $\alpha$-catenin, cells were fixed for $10 \mathrm{~min}$ at room temperature (RT) with $3 \%$ paraformaldehyde in PBS and treated for 5 min with 0.5\% Triton-X 100 (Sigma-Aldrich) in PBS on ice. For ZO-1, claudin-1 and occludin detection, cells were fixed with ethanol (for $30 \mathrm{~min}$ at $4^{\circ} \mathrm{C}$ ) and then with acetone (for $3 \mathrm{~min}$ at $-20^{\circ} \mathrm{C}$ ). Incubations with the primary and the secondary antibody were carried out for $1 \mathrm{~h}$ each at RT. Nuclei were stained with $0.2 \mu \mathrm{g} / \mathrm{ml}$ DAPI
A

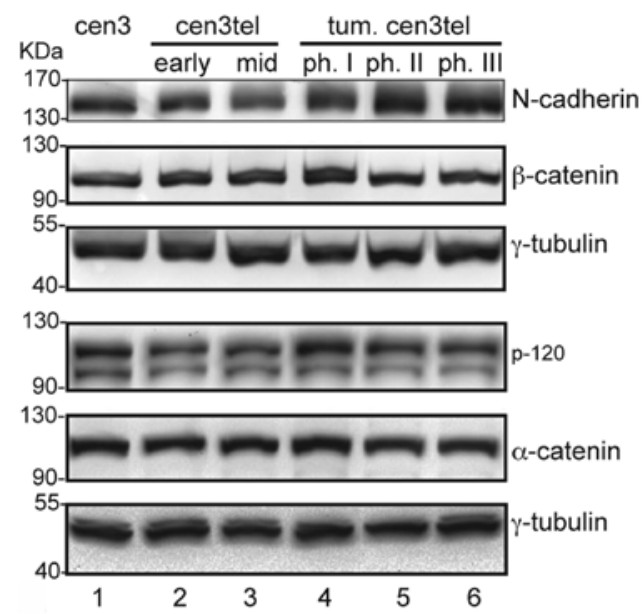

B

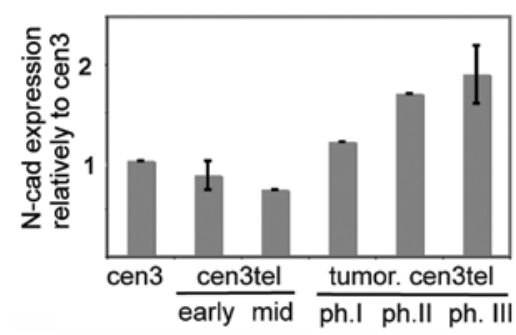

Figure 1. Expression of $\mathrm{N}$-cadherin, $\alpha$-catenin, $\mathrm{p}-120$ and $\beta$-catenin in cen 3 primary fibroblasts and in cen 3 tel at different stages of transformation. (A) Protein expression was analyzed by Western blot analysis using antibodies specific for each protein; $\gamma$-tubulin was used as loading control. (B) $\mathrm{N}$-cadherin expression in cen3tel cells relatively to cen 3 cells; results derive from two independent experiments (bar, standard deviation).

(4',6-diamidino-2-phenylindole) in PBS for $10 \mathrm{~min}$. Slides were analyzed using a Leica TCS SP2 confocal laser microscope equipped with a x63 objective (NA 1.25) or with an optical microscope Olympus IX71 equipped with a x60 objective (NA 1.25). Images were acquired using the Leica confocal software or with a digital camera Cool $\mathrm{SNAP}_{\mathrm{ES}}$ (Photometrics) using the MetaMorph software. Figures were assembled using Adobe Photoshop and Adobe Illustrator.

Western blot analysis. Whole-cell lysates were prepared using the RIPA buffer (1\% Nonidet P40, $50 \mathrm{mM}$ Tris-HCl pH 8.0, $150 \mathrm{mM} \mathrm{NaCl}, 0.1 \%$ SDS, $0.1 \%$ DOC, $1 \mathrm{X}$ protease inhibitor cocktail (Roche) and $0.2 \% \mathrm{Na}_{3} \mathrm{VO}_{4}$ ). For each sample $20 \mu \mathrm{g}$ of proteins were loaded on denaturing acrylamide gels. Chemiluminescent (Pierce) assays were used to detect the secondary antibody signal.

\section{Results}

To test whether neoplastic transformation and changes in cell morphology were associated with variations in the expression and/or subcellular localization of adhesion molecules, we analyzed the expression of $\mathrm{N}$-cadherin, $\alpha$-catenin, $\mathrm{p}-120$ and $\beta$-catenin in cen3tel cells at different stages of propagation.

Western blot analysis revealed an up to about 2 -fold increase in N-cadherin expression in tumorigenic cen3tel cells (Fig. 1A lanes 4-6) compared to parental cen3 fibroblasts, early and mid 

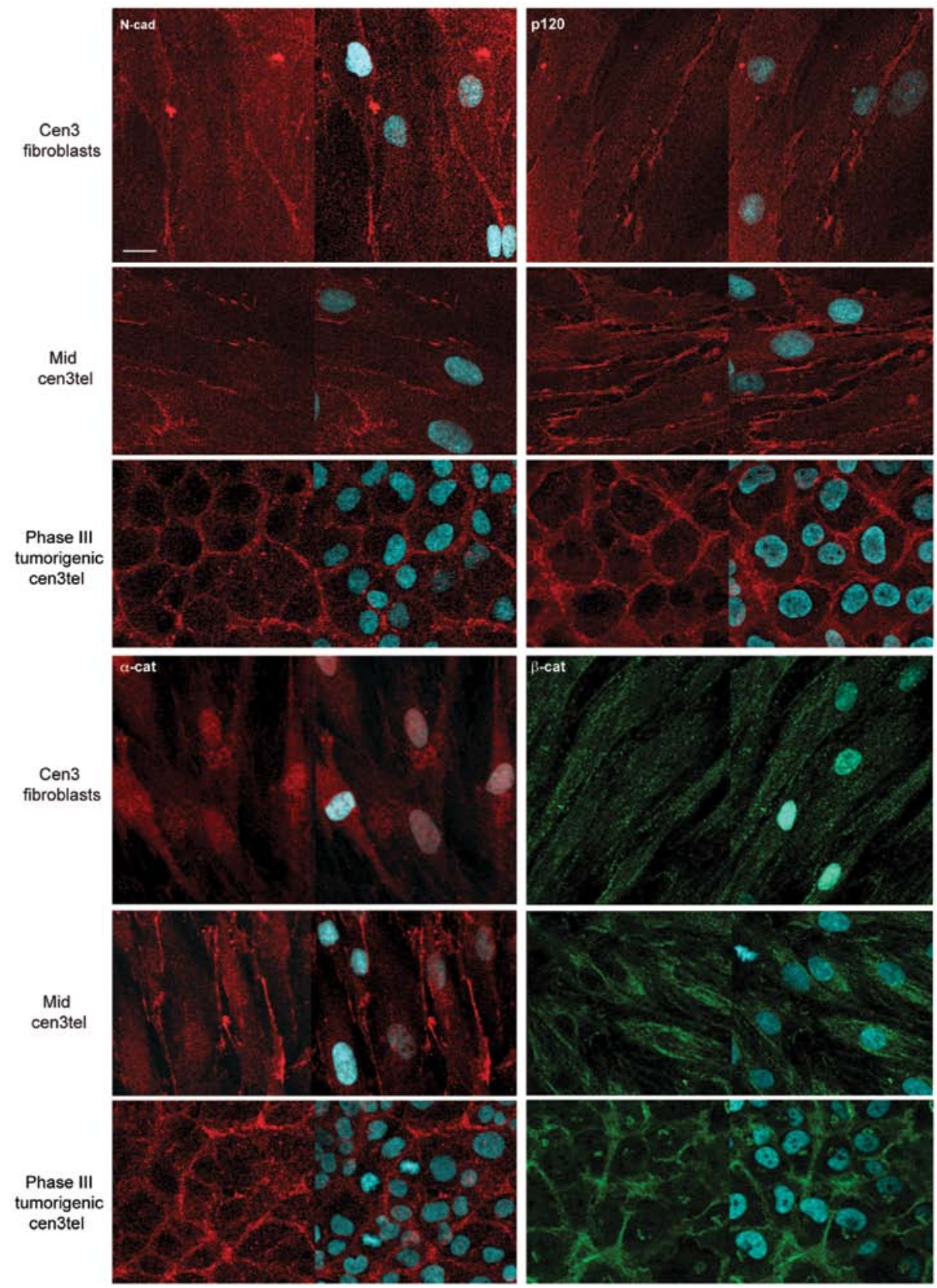

Figure 2. Subcellular localization of N-cadherin, $\alpha$-catenin, p-120 and $\beta$-catenin in cen 3 primary fibroblasts, mid and phase III tumorigenic cen3tel cells, by IIF. On the left side of each panel, staining due to the primary antibody against each specific protein (as indicated in the figure insets) is shown; on the right panel, merging between antibody and DAPI nuclear staining. Images were taken using a confocal microscope (x63 objective, bar $20 \mu \mathrm{m}$ ).

cen3tel cells (Fig. 1A lanes 1-3), and similar levels of expression of the three catenin molecules in cen3 fibroblasts and in cen3tel cells at different stages of propagation (Fig. 1). Indirect immunofluorescence (IIF) showed a re-localization of the four adhesion molecules in transformed cells (Fig. 2). In primary fibroblasts (Fig. 2) and early cen3tel cells (data not shown), $\mathrm{N}$-cadherin and p-120 were distributed in the cytoplasm with a weak staining around the cells along the plasma membrane; $\alpha$-catenin showed an intense cytoplasmic and nuclear staining, while $\beta$-catenin was only cytoplasmic (Fig. 2). In mid cen3tel cells (Fig. 2), the membrane signal started to increase, but the cytoplasmic one, and the nuclear one for $\alpha$-catenin, were still evident. In tumorigenic cells, the four molecules were mainly localized at the plasma membrane (Fig. 2, phase III tumori- genic cen3tel cells are shown as example); $\beta$-catenin showed also a perinuclear localization, suggesting that the protein did not translocate completely from the Golgi to the membrane. In tumorigenic cells, staining with each antibody revealed a honeycomb organization of the cell monolayer, suggesting that defined junctions were formed between cells.

The epithelial-like appearance of cen3tel cell monolayer prompted us to investigate the possibility that cen3tel cells had undergone MET. As markers for MET, we analyzed the expression of vimentin and cytokeratins, which are the intermediate filaments found in mesenchymal and epithelial cells, respectively, and of proteins involved in tight junction formation (claudin-1, occludin and ZO-1), which are typical of epithelial cells. 
A
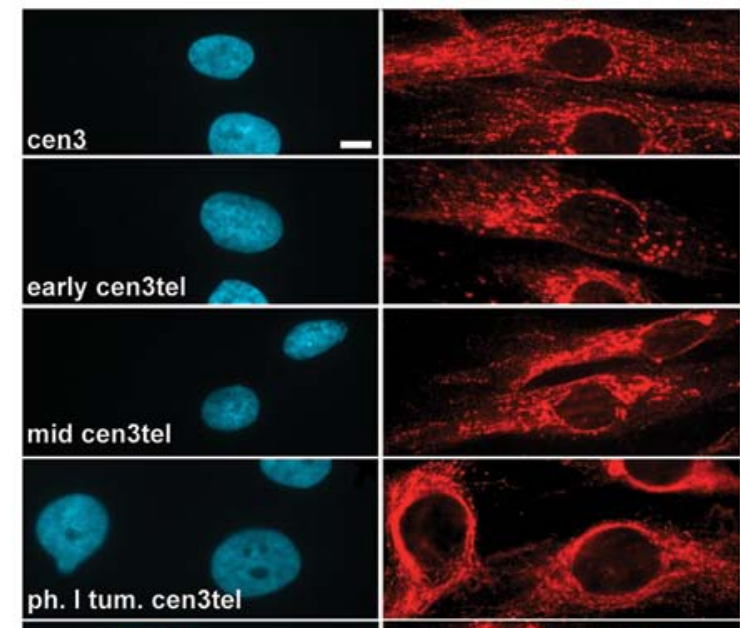

ph. I tum. cen3tel
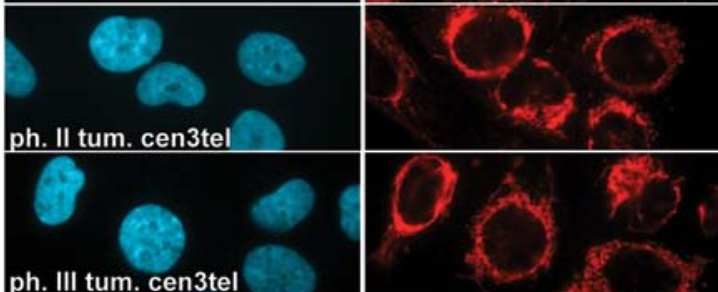

B

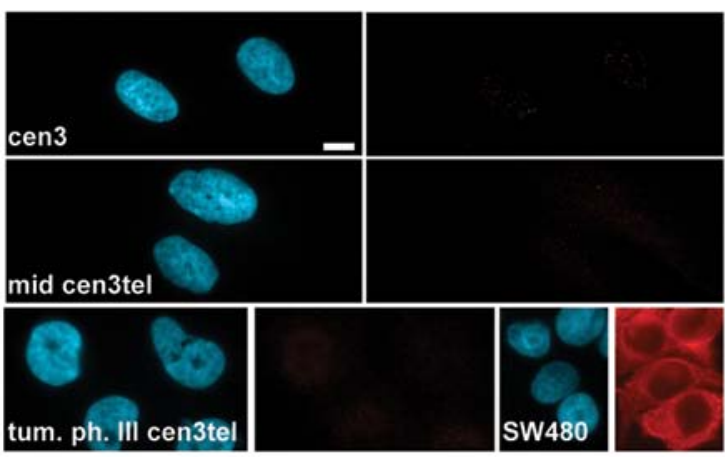

Figure 3. Analysis of vimentin subcellular distribution (A) and cytokeratin expression (B) in cen3 fibroblasts and cen3tel cells at different stages of transformation by IIF. On the left side panels, DAPI staining of cell nuclei on the right side panels, staining with the anti-vimentin antibody (A) or the anti-pan-cytokeratin antibody (B). SW480 epithelial cells were used as positive control for cytokeratin expression. Images were taken using an optical microscope (x60 objective, bar $10 \mu \mathrm{m}$ ).

As shown in Fig. 3, vimentin was expressed at all the stages of cen3tel propagation, but with a different subcellular distribution. In fact, in the more elongated primary fibroblasts as well as in early and mid cen3tel cells (Fig. 3A), vimentin was distributed along the cytoplasm, while, in polygonal tumorigenic cells, it was mainly localized around the nucleus (Fig. 3A). In contrast, we did not observe cytokeratin expression at any stage of propagation, indicating that tumorigenic cen3tel cells had not switched to the expression of the epithelial intermediate filaments (as an example, the results of the indirect immunofluorescence performed on cen 3 primary fibroblasts, mid and phase III tumorigenic cells are shown in Fig. 3B).

Tight junctions are formed at the apical surface of the lateral membrane of epithelial cells. They are composed of transmembrane proteins, such as those belonging to the claudin and occludin families, which are linked to the cytoskeleton through adaptor proteins belonging to the zona occludens
A

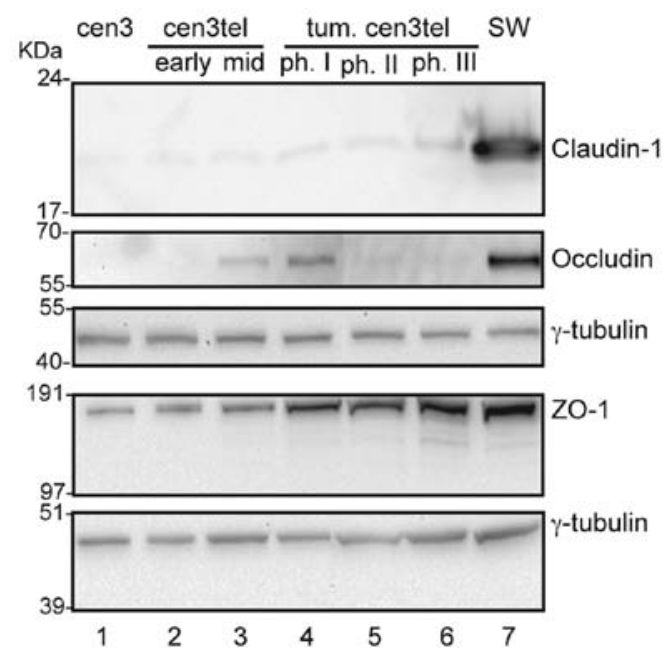

B

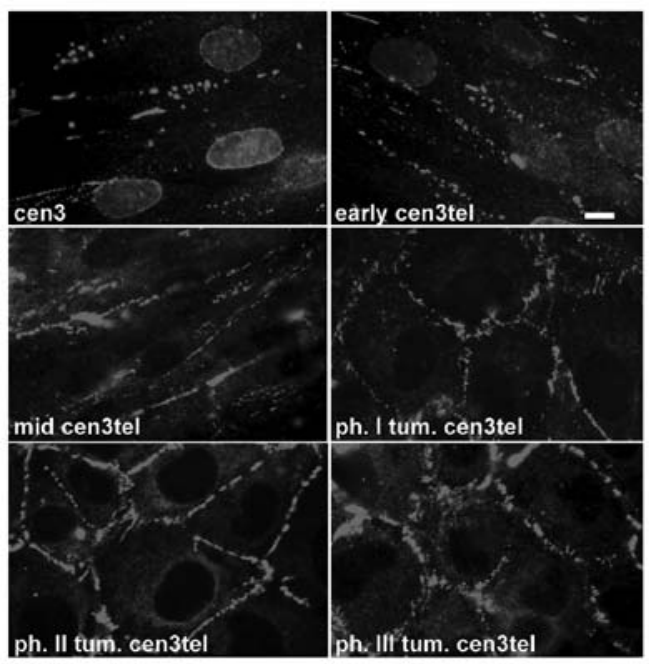

Figure 4. Expression of tight junction proteins in cen3 primary fibroblasts and cen3tel cells at different stages of transformation. (A) Western blot analysis of claudin-1, occludin and ZO-1 expression in total protein extracts using antibodies specific for each protein; $\gamma$-tubulin was used as loading control. SW480 (SW in the Figure) epithelial cells were used as positive control. (B) Analysis of ZO-1 subcellular localization by IIF. Images were taken using an optical microscope (x60 objective, bar $10 \mu \mathrm{m}$ ).

(ZO) family (13). In non-epithelial cells, $\mathrm{ZO}-1$ is localized at the cadherin based adhesion sites (14). Western blot analysis of claudin-1, occludin and ZO-1 expression (Fig. 4A) disclosed different expression patterns for the different proteins. Anticlaudin-1 antibody revealed a faint band in cen3 fibroblasts and in cen3tel cells at different PDs, whose intensity was much lower compared to that observed in the SW480 tumor cells, which were used as epithelial origin control cells (Fig. 4A). Occludin was undetectable in cells at the different stages of propagation, while showed a peak of expression in mid cen3tel cells and phase I tumorigenic cells (Fig. 4A, lanes 3 and 4). However, the expression level was lower than in epithelial SW480 cells (Fig. 4A, lane 7). Finally, ZO-1 presented increasing expression levels starting from mid cen3tel cells, with a net increase in tumorigenic cells (Fig. 4A, lanes 4-6 vs. 1-3). The expression level in tumorigenic cells was comparable to that observed in SW480 cells (Fig. 4A, lanes 4-6 vs. 7). By immunofluorescence, we found that ZO-1 presented a 
membrane localization that became more and more defined with progression towards transformation (Fig. 4B). In fact, in cen 3 fibroblasts and in early cen3tel cells, the protein signal was scattered along the membrane, in mid cen3tel cells it was more continuous and in tumorigenic cells it clearly defined cell boundaries. In primary cen3tel cells, as well as in other primary fibroblast strains (data not shown) and in early cen3tel cells, ZO-1 was also localized in the nucleus (Fig. 4B).

\section{Discussion}

In this study, we show that cellular localization of proteins involved in adherens junction formation varies during malignant transformation of human fibroblasts. In primary fibroblasts and early cen3tel cells, adhesion proteins were mostly localized in the cytoplasm, while membrane localization started to become more defined in mid cen3tel cells, which were at the initial phases of transformation and had lost contact inhibition. In tumorigenic cen3tel cells, in parallel to changes in morphology and actin distribution, and formation of high-density monolayers, $\mathrm{N}$-cadherin, $\alpha$-catenin, $\beta$-catenin and p120 acquired a clear-cut membrane localization. Thus, during neoplastic transformation of human fibroblasts we found that cell-contacts were more defined in tumorigenic cells than in non-tumorigenic ones. It was shown that loss of contact inhibition characterizing transformed cells could be associated with a deregulation of cellular adhesion systems (15); our results suggest that tumorigenic fibroblasts can reach a high cellular density and form strong cell-cell contacts without growth inhibition. $\mathrm{N}$-cadherin showed an about 2-fold increased expression in tumorigenic cen3tel cells; N-cadherin is known to promote a dynamic adhesion state in tumor cells together with increased proliferation in some tumors $(16,17)$, its enhanced levels might have contributed to the acquisition of the invasive capacity by tumorigenic cen3tel cells and of the metastatic potential by phase III tumorigenic cells.

In mesenchymal tumors, e.g. in synovial sarcomas, the presence of epithelial-like cells can be associated with the expression of epithelial markers, such as cytokeratins, or tight junction proteins. In the glands of biphasic synovial sarcomas, true, well-organized tight junctions were recognized (6). In our cellular system, variations in adhesion molecule subcellular localization and morphological changes were not associated with a switch in the expression of the intermediate filaments, from vimentin to cytokeratins, or by the expression of tight junction proteins suggestive of mature tight junction formation, which could be indicative of MET occurrence during neoplastic transformation. We did find a transient expression of occludin in cells at the transition point from 'normal' cells and tumorigenic ones (mid cen3tel cells) or in cells at the early stage of tumorigenesis (cen3tel cells of tumorigenic phase I), but this expression greatly decreased at later stages. It might be that cen3tel had started MET during the initial phases of transformation, but the transition was not completed, possibly because it did not confer any selective advantage to the cells. However, it could also be hypothesized that occludin expression had a role in the transformation process and was not required anymore when transformation was completed.

A role in the control of cell proliferation and gene expression was actually shown for tight junction proteins (18). ZO-1 can control gene expression by binding to transcription factors, such as ZONAB (ZO-1 associated nucleic acid-binding protein) $(19,20)$. In addition, $\mathrm{ZO}-1$ was reported to accumulate transiently in the nucleus of proliferating epithelial cells, where it could regulate cellular processes (21). In the cen3tel cellular system, we detected ZO-1 nuclear staining in primary fibroblasts and in early cen3tel cells, that is in cells that were not transformed. In these cells, ZO-1 levels were lower compared to those found in transformed cells, suggesting that nuclear localization did not depend on the presence of high levels of the endogenous protein. In epithelial cells, ZO-1 nuclear localization seemed to be inversely related to the extent and/ or maturity of cell-cell contacts. This could hold true also for cells of mesenchymal origin: in primary fibroblasts and in early cen3tel cells, which show contact inhibition and a lower degree of cell-cell contacts, ZO-1 was also localized in the nucleus, in cells at the early stages of transformation or neoplastically transformed, which can reach a high cellular density, ZO-1 became mainly localized at the plasma membrane. ZO-1 membrane localization in cen3tel cells and the increased levels paralleling the increasing levels of $\mathrm{N}$-cadherin fit with the knowledge that ZO-1 also participates in adherens junction formation in mesenchymal cells (22).

Analogously to that observed for ZO-1 in non-tumorigenic cen3tel cells as well as in other normal fibroblast strains (data not shown), we found that $\alpha$-catenin showed both nuclear and cytoplasmic localization, while in tumorigenic cells it was mainly localized at the plasma membrane. In some colon cancer cell lines, nuclear localization of $\alpha$-catenin was detected when cells were dispersed rather than confluent and it was proposed that it was related to the lack of a strong connection between adjacent cells (23). This explanation can be true for $\alpha$-catenin nuclear localization in non-tumorigenic cen3tel cells. Moreover, evidence was reported that nuclear $\alpha$-catenin can play a tumor suppressive role in colon cancer cells by preventing the interaction between the Tcf $/ \beta$-catenin complex and its DNA target, and thus the activation of cancer genes (24). Similarly, in normal cells, nuclear $\alpha$-catenin could interact with transcription factors regulating the expression of cancer genes.

In conclusion, studying neoplastic transformation of human fibroblasts, from normal up to tumorigenic cells, we found that tumorigenic cells underwent a change in cell morphology, with actin redistribution from stress fibers to circular bundles and, following the loss of contact inhibition, they formed high density cell cultures $(7,9)$. We have shown here that these changes are associated with membrane re-localization of different adhesion molecules and increased $\mathrm{N}$-cadherin and ZO-1 expression levels and are independent of a transition from the mesenchymal to the epithelial phenotype. Our results suggest that deregulation of cell-cell adhesion is not required for the loss of growth cell contact inhibition. Moreover, we found that both ZO-1 and $\alpha$-catenin showed nuclear localization in untransformed cells, suggesting that these molecules can play additional functions in the cells besides cell-junction formation.

\section{Acknowledgements}

This study was supported by Fondazione Cariplo (Grant 20060734). C.B. is a doctoral fellow of the University of Pavia 
(Dottorato in Scienze Genetiche e Biomolecolari). We are very grateful to to Dr Patrizia Vaghi (University of Pavia, Italy) for confocal microscope analysis.

\section{References}

1. Halbleib JM and Nelson WJ: Cadherins in development: cell adhesion, sorting, and tissue morphogenesis. Genes Dev 20: 3199-3214, 2006

2. Pokutta S and Weis WI: Structure and mechanism of cadherins and catenins in cell-cell contacts. Annu Rev Cell Dev Biol 23: 237-261, 2007.

3. Brembeck FH, Rosario M and Birchmeier W: Balancing cell adhesion and Wnt signaling, the key role of beta-catenin. Curr Opin Genet Dev 16: 51-59, 2006.

4. Polyak K and Weinberg RA: Transitions between epithelial and mesenchymal states: acquisition of malignant and stem cell traits. Nat Rev Cancer 9: 265-273, 2009.

5. Deyrup AT and Montag AG: Epithelioid and epithelial neoplasms of bone. Arch Pathol Lab Med 131: 205-216, 2007.

6. Billings SD, Walsh SV, Fisher C, Nusrat A, Weiss SW and Folpe AL: Aberrant expression of tight junction-related proteins ZO-1, claudin-1 and occludin in synovial sarcoma: an immunohistochemical study with ultrastructural correlation. Mod Patho 17: 141-149, 2004.

7. Belgiovine C, Frapolli R, Bonezzi K, Chiodi I, Favero F, MelloGrand M, Dei Tos AP, Giulotto E, Taraboletti G, D'Incalci M and Mondello C: Reduced expression of the ROCK inhibitor Rnd3 is associated with increased invasiveness and metastatic potential in mesenchymal tumor cells. PLoS One 5: e14154, 2010.

8. Mondello C, Chiesa M, Rebuzzini P, Zongaro S, Verri A, Colombo T, Giulotto E, D'Incalci M, Franceschi C and Nuzzo F: Karyotype instability and anchorage-independent growth in telomerase-immortalized fibroblasts from two centenarian individuals. Biochem Biophys Res Commun 308: 914-921, 2003.

9. Zongaro S, de Stanchina E, Colombo T, D'Incalci M, Giulotto E and Mondello C: Stepwise neoplastic transformation of a telomerase immortalized fibroblast cell line. Cancer Res 65 11411-11418, 2005.

10. Pankova K, Rosel D, Novotny M and Brabek J: The molecular mechanisms of transition between mesenchymal and amoeboid invasiveness in tumor cells. Cell Mol Life Sci 67: 63-71, 2010.

11. Sahai E: Mechanisms of cancer cell invasion. Curr Opin Genet Dev 15: 87-96, 2005.
12. Wolf K and Friedl P: Molecular mechanisms of cancer cell invasion and plasticity. Br J Dermatol 154 (Suppl 1): 11-15, 2006.

13. Balda MS and Matter K: Tight junctions at a glance. J Cell Sci 121: 3677-3682, 2008

14. Itoh M, Nagafuchi A, Yonemura S, Kitani-Yasuda T, Tsukita S and Tsukita S: The $220-\mathrm{kD}$ protein colocalizing with cadherins in non-epithelial cells is identical to ZO-1, a tight junction-associated protein in epithelial cells: cDNA cloning and immunoelectron microscopy. J Cell Biol 121: 491-502, 1993.

15. Ho AT, Voura EB, Soloway PD, Watson KL and Khokha R: MMP inhibitors augment fibroblast adhesion through stabilization of focal adhesion contacts and up-regulation of cadherin function. J Biol Chem 276: 40215-40224, 2001.

16. Cavallaro U and Dejana E: Adhesion molecule signalling: not always a sticky business. Nat Rev Mol Cell Biol 12: 189-197, 2011.

17. Tran NL, Adams DG, Vaillancourt RR and Heimark RL: Signal transduction from $\mathrm{N}$-cadherin increases Bcl-2. Regulation of the phosphatidylinositol 3-kinase/Akt pathway by homophilic adhesion and actin cytoskeletal organization. J Biol Chem 277: 32905-32914, 2002.

18. Balda MS and Matter K: Tight junctions and the regulation of gene expression. Biochim Biophys Acta 1788: 761-767, 2009.

19. Balda MS and Matter K: The tight junction protein ZO-1 and an interacting transcription factor regulate ErbB-2 expression. EMBO J 19: 2024-2033, 2000.

20. BauerH,Zweimueller-Mayer J, Steinbacher P,Lametschwandtner A and Bauer HC: The dual role of zonula occludens ( $\mathrm{ZO})$ proteins. J Biomed Biotechnol 2010: 402593, 2010.

21. Gottardi CJ, Arpin M, Fanning AS and Louvard D: The junctionassociated protein, zonula occludens-1, localizes to the nucleus before the maturation and during the remodeling of cell-cell contacts. Proc Natl Acad Sci USA 93: 10779-10784, 1996.

22. Fanning AS and Anderson JM: Zonula occludens-1 and -2 are cytosolic scaffolds that regulate the assembly of cellular junctions. Ann N Y Acad Sci 1165: 113-120, 2009.

23. El-Bahrawy M, Talbot I, Poulsom R and Alison M: Variable nuclear localization of alpha-catenin in colorectal carcinoma. Lab Invest 82: 1167-1174, 2002.

24. Giannini AL, Vivanco M and Kypta RM: alpha-catenin inhibits beta-catenin signaling by preventing formation of a beta-catenin*T-cell factor*DNA complex. J Biol Chem 275: 21883-21888, 2000. 